\title{
Multi-period stochastic lateral transshipment problem for rental products
}

\section{Koji Aragane, Tomoki Fukuba and Takayuki Shiina*}

\author{
Department of Industrial and Management System Engineering, \\ Waseda University, \\ Tokyo, Japan \\ Email: argnkj@suou.waseda.jp \\ Email: tomohikari892@asagi.waseda.jp \\ Email: tshiina@waseda.jp \\ *Corresponding author
}

\begin{abstract}
In this study, we employed a multi-period stochastic programming model to examine the lateral transshipment problem of rental products and verified the effectiveness of this model under several assumptions about demand trends. The design and operation of multi-period supply chain networks is a large-scale optimisation problem under uncertainty. Lateral transshipment between bases is effective in improving service levels and in reducing inventory. In this study, we assume that the quantity of demand and length of rentals follow a probability distribution (since it deals with rental products). We construct a scenario tree to consider the uncertainty of rental products in multi-periods using the K-means method. Considering demand trends, we construct three types of scenario trees that represent flat, growth, and decline trends. We demonstrate the effectiveness of the model for each trend through numerical experimentation.
\end{abstract}

Keywords: stochastic programming model; lateral transshipment; rental product; supply chain; K-means method; scenario tree; multi-periods.

Reference to this paper should be made as follows: Aragane, K., Fukuba, T. and Shiina, T. (2021) 'Multi-period stochastic lateral transshipment problem for rental products', Asian J. Management Science and Applications, Vol. 6, No. 1, pp.32-48.

Biographical notes: Koji Aragane received his BE in Management System Engineering from Waseda University, Tokyo Japan, in 2019. His research interests include supply chain management.

Tomoki Fukuba received his BE and ME in Management System Engineering from Waseda University, Tokyo, Japan, in 2018 and 2020, respectively. His research interests include mathematical optimisation.

Takayuki Shiina is a Professor at the Department of Industrial and Management Systems Science, School of Creative Science and Engineering, Waseda University, Japan. He received his BE, ME, and Doctor of Engineering from Waseda University. He has been studying mathematical programming at the Central Research Institute of the Electric Power 
Industry (Tokyo, 1991-2008), Northwestern University (Evanston, Illinois, 2000-2001), and Chiba Institute of Technology (Narashino, Chiba, Janan, 2008-2015). His main areas of interest are stochastic programming and integer programming. He was awarded the best paper prize with Professor John R. Birge (University of Chicago) from the Japan Society of Industrial and Applied Mathematics in 2005.

This paper is a revised and expanded version of a paper entitled 'The multi-period stochastic programming model of a lateral transshipment problem considering for rentals and returns' presented at the 2019 Asian Conference of Management Science \& Applications (ACMSA2019), Penglai, Shandong, China, 11-14 October 2019.

\section{Introduction}

The design and operation of multi-period supply chain networks is a large-scale optimisation problem under uncertainty. To construct efficient supply chain networks, many studies have been conducted using stochastic programming. Companies aim to improve service levels as well as reduce the cost of the entire supply chain, including the costs of inventory and transportation. However, to improve service levels, a large inventory is required. Lateral transshipment between bases is effective as a strategy to improve service levels while keeping inventory costs low. Through lateral transshipment, it is possible to avoid stockout while suppressing upstream orders.

We call the rental products of a prefabricated house a unit house, which is used for construction site offices, rental warehouses, event facilities, and so on. The demand for unit houses in Japan is increasing due to big projects such as the Tokyo Olympics. In general, buildings are constructed after materials are brought to the relevant location. However, unit houses are produced in a factory and then transported to the site, which is a time-saving measure. Because the operation of unit houses is not a construction issue but a production issue, it is necessary to design a supply chain model.

Patterson et al. (2011) conducted a literature review and categorised previous studies of lateral transshipment. Lateral transshipment models are categorised into two categories: those that occur at a predetermined time before all demand is known, and those that can be done at any time to accommodate a potential shortage. These two types are called proactive transshipments and reactive transshipments. The review by Patterson et al. (2011) is comprehensive and should be consulted for previous research. However, most of the studies covered in their review are not based on mathematical optimisation models. Agrawal et al. (2004) considered a dynamic version of the classic problem of inventory allocation to retailers and how to correct inventory imbalances between retailers. They dealt with a dynamic rebalancing problem with two decisions: shipment timings and determining new retailer inventory levels, rather than taking a mathematical optimisation approach. Topan and van der Heijden (2020) studied operational spare parts planning for a multi-item two-echelon distribution system, taking into account real-time supply information within the system. In this study, a wide range of operational interventions, reactive, and proactive measures are investigated. These models included lateral transshipment between warehouses and urgent shipments from depots. Naderi et al. (2020) formulated the problem of a fast fashion retailer as a large 
mixed integer linear program and developed a Lagrangian relaxation with a primal-dual approach to find the upper bounds and a simulated annealing-based metaheuristic to find promising solutions. However, the models covered in these recent papers do not include fluctuations in customer demand.

Decision making under uncertainty involves potential risk. Stochastic programming (Birge, 1997; Birge and Louveaux, 1997; Kall and Wallace, 1994) deals with optimisation under uncertainty. A stochastic programming problem with recourse is referred to as a two-stage stochastic programming problem. In the first stage, a decision has to be made without complete information on random factors. After the value of random variables is observed, recourse action can be taken in the second stage. For the continuous stochastic programming problem with recourse, the L-shaped method (van Slyke and Wets, 1969) is widely used. The L-shaped method is used to solve stochastic programs having discrete decisions in the first stage (Laporte and Louveaux, 1993).

Kaut and Wallace (2007) presented an overview of the most important scenario-generation methods. They classified these methods into sampling-based methods and path-based methods. Sampling-based methods generate scenario trees, and path-based methods generate scenario fans. If the uncertainty is represented by a multivariate continuous distribution or a discrete distribution with too many outcomes, there is a need to reduce the number of possible scenarios. For this purpose, Høyland and Wallace (2001) presented the moment matching method based on nonlinear programming that can be used to generate a limited number of discrete results that meet specified statistical properties. Gülpınar et al. (2004) studied randomly generated scenarios as well as several variants of the moment matching procedure and reported on the implementation results of these methods. In $\mathrm{Hu}$ and $\mathrm{Hu}$ (2016), a lot-sizing and scheduling problem with sequence dependent setups is addressed, and the moment matching method is applied to scenario generation.

However, in order to apply the moment matching method, a theoretical distribution must be assumed. For moment matching, large-scale nonlinear programming problems are required. Möller et al. (2004) described the backward and forward scenario tree generation methods based on upper bounds for two relevant ingredients of the stability estimate. Sutiené et al. (2010) presented an approach to generate the multistage multidimensional scenario tree out of a set of scenario fans. For this purpose, the multistage K-means clustering algorithm was developed. $\mathrm{Xu}$ et al. (2012) developed new approaches by combining simulation, K-means clustering, and moment matching skills to generate the general multi-stage scenario tree.

In addition to lateral transshipment, when designing logistics networks, deciding which facilities to use is a crucial supply chain decision. Maintenance of facilities and relocation must be considered in the use of existing and new facilities when reorganising logistics networks. Restructuring the network requires significant investment. Therefore, the best decisions can be taken from a long-term perspective. However, optimising the network is difficult because customer demand is unpredictable. Decisions regarding facility relocation must be made at a strategic level.

Melachrinoudis and Min (2000) proposed a deterministic model that considers the relocation of one facility. Melo et al. (2005) proposed a fixed demand multiperiod model with multiple reorganisation goals. Melachrinoudis and Min (2007) reorganised a network using real data as a case study. Kiya and Davoudpour (2012) proposed a 
model which considered the uncertainty of demand and transportation cost and applied the L-shaped method.

Shiina et al. (2014) proposed a stochastic model of a lateral transshipment problem. Through lateral transshipment between bases, it is possible to improve service levels while suppressing upstream orders. Combining the two policies of preventive and emergency lateral transshipment enables higher service levels to be achieved. The stochastic programming model is formulated with demand as a stochastic variable. The model is used to find an efficient solution for the problem.

Schildbach and Morari (2016) proposed scenario-based model predictive control (SCMPC) to manage a multi-period supply chain, which is a large-scale problem affected by uncertainty. In their paper, uncertain demand is completely satisfied by robust optimisation.

Lin (2009) proposed a stochastic single-source capacitated facility location problem. Facilities are selected to provide service to demand points with stochastic demand at the minimal total cost. The facilities have service level requirements formulated as chance constraints. For problems with Poisson demand, chance constraints have been proven to be equivalent to a solvable deterministic problem. For problems with normally distributed demand, chance constraints are equivalent to a deterministic mixed integer nonlinear programming problem. They proposed an approximate solution using a hybrid heuristic of Lagrangian relaxation.

A unique feature of this study is that it takes into consideration the fluctuation in demand for rental items and in returns. The above-mentioned scenario generation method considered fluctuations in demand only. In this study, we provide products to the demand for rental products. The length of the rental period for these products is defined as a random variable. There are few studies on the generation of scenario trees in consideration of such fluctuations in demand and in time to return. We present a method of generating a scenario tree in which the amount of rental and return are consistent, using the K-means method. In the conventional supply chain planning problem that considers recycling and reuse, the amount of demand and the amount of reuse (return) are treated as different problems. This study is suitable for realistic planning because it considers a scenario with fluctuation in the return volume related to the demand, which is more realistic than a scenario where the volume is static.

\section{Lateral transshipment problem considering rentals and returns}

\subsection{Problem description}

We aim to minimise the total cost of multi-period supply chain operation. The model of the supply chain network for rental products is shown in Figure 1. The network consists of facilities and customers in three echelons. The first echelon consists of production factories. If existing factories cannot satisfy demands, a new factory will be installed from among candidate sites. The second echelon consists of warehouses (bases). To satisfy demands from customers, bases place orders with factories and perform lateral transshipment between bases. Lead times of production and transportation are not considered in this paper. If bases do not satisfy the demands of customers, a penalty cost is incurred. The third echelon consists of customers, who receive supply from bases. Customers rent products from an individual base and return them to the same base. The 
returned products can be reused at a fixed rate. We assume that customer demand and the length of rental periods follow a probability distribution. As a result, the customer demand and return quantity follow a probability distribution. The procedure for scenario generation considering rentals and returns is shown in Section 3.

Figure 1 Schematic overview of supply chain network

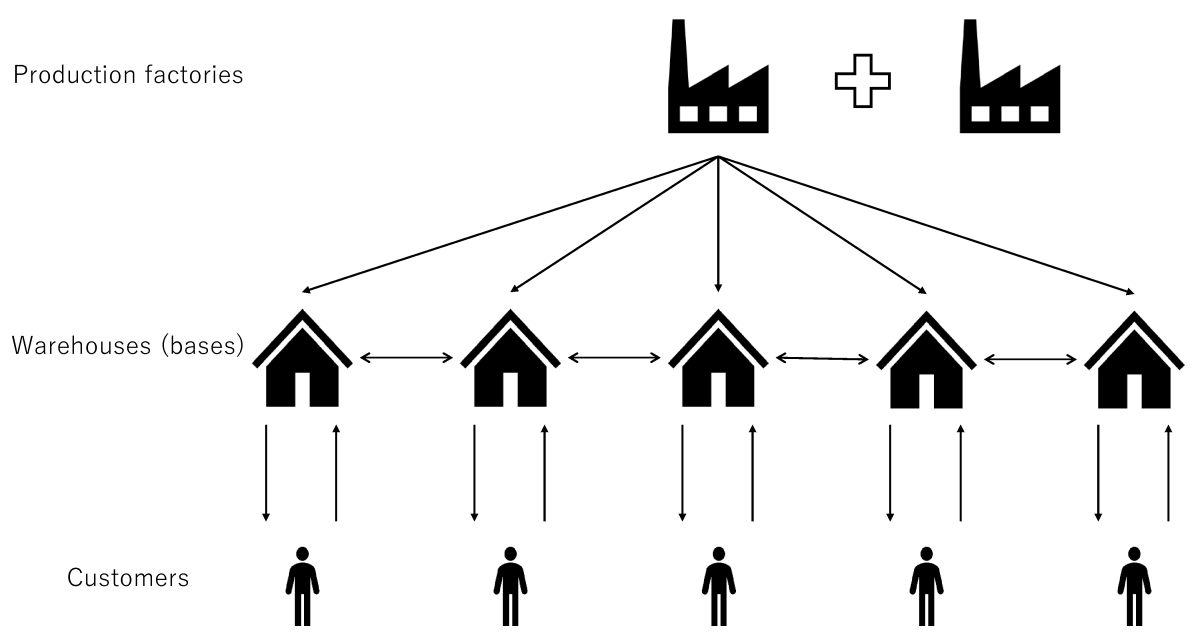

\subsection{Notations}

This section presents the mathematical formulation of a multi-period stochastic model for a lateral transshipment problem. It is assumed that rental quantity $\tilde{\xi}_{t}$ and return quantity $\tilde{\zeta}_{t}$ are defined in a known probability space and follow a finite discrete probability distribution. Let $\xi_{t}^{k}, \zeta_{t}^{k}$ be a realisation of random variables $\tilde{\xi}_{t}, \tilde{\zeta}_{t}$. The notation used in the formulation is as follows:

Sets

$E \quad$ the set of existing factories

$C \quad$ the set of potential factories

$I \quad$ the set of bases

$N_{i} \quad$ the set of customers belonging to base $i$

$K_{t} \quad$ the set of scenarios in period $t$

$T$ the set of periods. 


\section{Variables}

$w_{f t} \quad 0-1$ decision variable, 1 if the potential factory $f \in C$ is installed in period $t$, 0 otherwise

$y_{i t}^{k} \quad 0-1$ decision variable, 1 if stockout occurs at the base $i$ under scenario $k$ in period $t, 0$ otherwise

$s_{i t}^{k} \quad$ inventory quantity of base $i$ under scenario $k$ in period $t$

$z_{i t}^{k} \quad$ stockout quantity of base $i$ under scenario $k$ in period $t$

$o_{f i t}^{k} \quad$ order quantity of the base $i$ to factory $f$ under scenario $k$ in period $t$

$x_{i j t}^{k} \quad$ lateral transshipment quantity from base $i$ to base $j$ under scenario $k$ in period $t$.

\section{Parameters}

$\xi_{i t n}^{k} \quad$ rental quantity to customer $n$ belonging to base $i$ under scenario $k$ in period $t$

$\zeta_{i t n}^{k} \quad$ return quantity from customer $n$ belonging to base $i$ under scenario $k$ in period $t$

$F_{f t} \quad$ installation cost of potential factory $f \in C$ in the period $t$

$H_{i t}^{k} \quad$ inventory cost of base $i$ under scenario $k$ in period $t$

$P_{i t}^{k} \quad$ stockout cost of base $i$ in scenario $k$ of period $t$

$R_{f i t}^{k} \quad$ order cost of base $i$ to factory $f$ under scenario $k$ in period $t$

$L_{i j t}^{k} \quad$ lateral transshipment cost from base $i$ to base $j$ under scenario $k$ in period $t$

$\beta \quad$ reusable rate of returned products

$\gamma_{t} \quad$ service level requirement regarding stockout

$C A P_{f} \quad$ capacity of the factory $f \in E \cup C$

$p_{t}^{k} \quad$ occurrence probability of scenario $k$ in period $t$

$\alpha(k, t) \quad$ parent node number in period $t-1$ of scenario $k$ in period $t$.

We construct a scenario tree to represent the uncertainty in a multi-period timeframe. The scenario tree is shown in Figure 2 and is composed of one root node at the initial stage, which branches into a finite number of descendant nodes in every stage. Assuming that the number of branches is 2 , the number of nodes $K_{t}$ at stage $t$ is $2^{t}$. The predecessor of scenario $k$ in period $t$ is denoted by $\alpha(k, t)$. These relationships are illustrated in Figure 3. 
Figure 2 A scenario tree

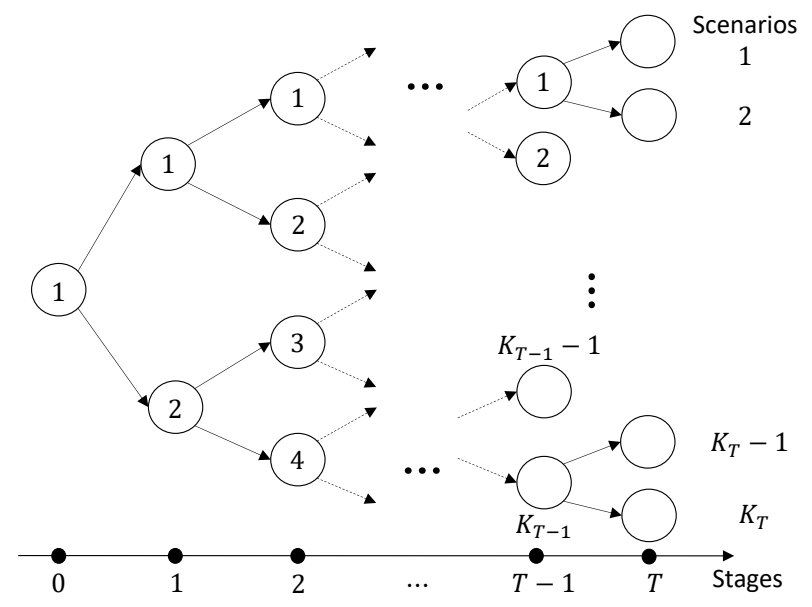

Figure 3 Scenarios and predecessor of nodes

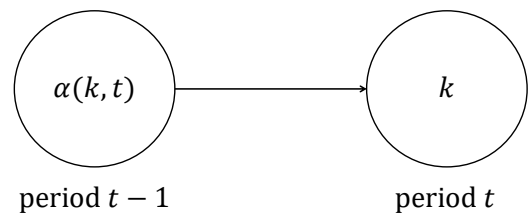

\subsection{Formulation}

The proposed problem is formulated as the multi-period stochastic problem using scenarios (Shiina and Birge, 2002; Shiina, 2015). The problem is shown as follows.

$$
\begin{aligned}
& \min \sum_{t \in T} \sum_{f \in C} F_{f} w_{f t}+\sum_{t \in T} \sum_{k \in K_{t}}\left(\sum_{i \in I} \sum_{j \neq i} p_{t}^{k} L_{i j t}^{k} x_{i j t}^{k}+\sum_{f \in E \cup C} \sum_{i \in I} p_{t}^{k} R_{f i t}^{k} o_{f i t}^{k}\right. \\
& \left.+\sum_{i \in I} p_{t}^{k} H_{i t}^{k} s_{i t}^{k}+\sum_{i \in I} p_{t}^{k} P_{i t}^{k} z_{i t}^{k}\right)
\end{aligned}
$$

s.t.

$$
\begin{aligned}
& \sum_{t \in T} \sum_{f \in C} w_{f t} \leq 1 \\
& \sum_{i \in I} o_{f i t}^{k} \leq C A P_{f} \quad \forall f \in E, \forall t \in T, \forall k \in K_{t} \\
& \sum_{i \in I} o_{f i t}^{k} \leq C A P_{f} \sum_{\tau=1}^{t} w_{f \tau} \quad \forall f \in C, \forall t \in T, \forall k \in K_{t}
\end{aligned}
$$




$$
\begin{aligned}
& s_{i t}^{k}-z_{i t}^{k}=s_{i t-1}^{\alpha(k, t)}+\sum_{f \in E \cup C} o_{f i t}^{k}+\sum_{j \in I} x_{j i t}^{k}-\sum_{j \in I} x_{i j t}^{k} \\
& \quad-\sum_{n \in N_{i}} \xi_{i t n}^{k}+\beta \sum_{n \in N_{i}} \zeta_{i t n}^{k} \quad \forall i \in I, \forall t \in T, \forall k \in K_{t} \\
& \sum_{j \in I} x_{i j t}^{k} \leq s_{i t-1}^{\alpha(k, t)} \quad \forall i \in I, \forall t \in T, \forall k \in K_{t} \\
& s_{i t-1}^{\alpha(k, t)}+\sum_{f \in E \cup C} o_{f i t}^{k}+\sum_{j \in I} x_{j i t}^{k}-\sum_{j \in I} x_{i j t}^{k} \\
& -\sum_{n \in N_{i}} \xi_{i t n}^{k}+\beta \sum_{n \in N_{i}} \zeta_{i t n}^{k}+M y_{i t}^{k} \geq 0 \quad \forall i \in I, \forall t \in T, \forall k \in K_{t} \\
& s_{i t-1}^{\alpha(k, t)}+\sum_{f \in E \cup C} o_{f i t}^{k}+\sum_{j \in I} x_{j i t}^{k}-\sum_{j \in I} x_{i j t}^{k} \\
& -\sum_{n \in N_{i}} \xi_{i t n}^{k}+\beta \sum_{n \in N_{i}} \zeta_{i t n}^{k}+M y_{i t}^{k} \leq M-\varepsilon \quad \forall i \in I, \forall t \in T, \forall k \in K_{t} \\
& \frac{\sum_{k \in K_{t}} \sum_{i \in I} y_{i t}^{k} \leq K_{t}}{\left|K_{t}\right| I \mid} \leq \forall t \in T \\
& w_{f}, u_{f i t}^{k}, y_{i t}^{k} \in\{0,1\}, \quad \forall f \in E, \forall i \in I, \forall t \in T, \forall k \in K_{t} \\
& s_{i t}^{k}, z_{i t}^{k}, o_{f i t}^{k}, x_{i j t}^{k} \geq 0 \\
& \forall f \in E, \forall i \in I, \forall t \in T, \forall k \in K_{t}
\end{aligned}
$$

The objective function (1) minimises the sum of the expected values of installation, lateral transshipment, order, inventory, and stockout costs. Constraint (2) ensures that there is at most one installed potential factory. Constraint (3) represents the maximum values of order quantity for an existing factory. Constraint (4) represents the maximum values of order quantity for a potential factory. Constraint (5) represents the conservation of inventory quantity at the base. The equation $s_{i t}^{k} \times z_{i t}^{k}=0$ is satisfied at the optimal solution. Constraint (6) ensures that the lateral transshipment quantity must be less than or equal to the last period's inventory quantity. Constraints (7)-(9) are related to stockout. We introduce a sufficiently positive large number $M$ and $y_{i t}^{k}$ to express the logical condition of whether stockout occurs at the base. Constraint (7) ensures $y_{i t}^{k}=1$ when stockout occurs. Constraint (8) ensures $y_{i t}^{k}=0$ when stockout does not occur. Constraint (9) ensures the maximum value of the stockout rate. Constraint (10) represents the decision variables, which are 0-1 variables. Constraint (11) ensures the decision variables are non-negative.

\section{Scenario generation of rentals and returns}

\subsection{The procedure of scenario generation}

We represent the uncertainties of rental and return quantities using a scenario tree. We assume that customers rent products once and return them together in the planning period. Rental products in the last period are not returned within the period. First, 
a scenario fan is generated considering these assumptions. The rental quantity $\tilde{\xi}_{t}$ in period $t$ follows a normal distribution with a mean of 100 and variance of 10 . Such assumptions are often made in both natural phenomena and social models. The length of the rental $\tilde{\tau}_{t}$ for the products rented in period $t$ follows a discrete uniform distribution with interval $[1, T-t]$. The return quantity $\zeta_{t}^{s}$ in period $t$ under scenario $s$ is defined to satisfy $\zeta_{t+\tau_{t}^{s}}^{s}=\xi_{t}^{s}$. This is because the amount $\tilde{\zeta}$ was set to be returned within the planning period for the lending amount $\tilde{\xi}$. Stochastic programming models generally do not consider infinite periods, but address decision making within finite periods, so it is assumed that lending and returning will be done within the planning period. Next, a scenario tree is generated out of the scenario fan by adapting the K-means method (Šutienè et al., 2010; Xu et al., 2012) considering only rental quantity. As a result, the rental and return quantities cannot be consistent. Therefore, it is necessary to modify the return quantity in the scenario tree according to the integrated rental quantity.

\subsection{K-means method for multi-stage problem}

Šutienė et al. (2010) proposed the multi-stage K-means clustering method, which is used to generate a scenario tree out of a scenario fan. The scenario fan is defined as the realisation value of the random variable from the first stage to the last stage as one sample path. The typical scenario fan is described in Figure 4. It consists of a large number of independent data paths. Problems with a scenario fan become large-scale optimisation problems.

Figure 4 A scenario fan

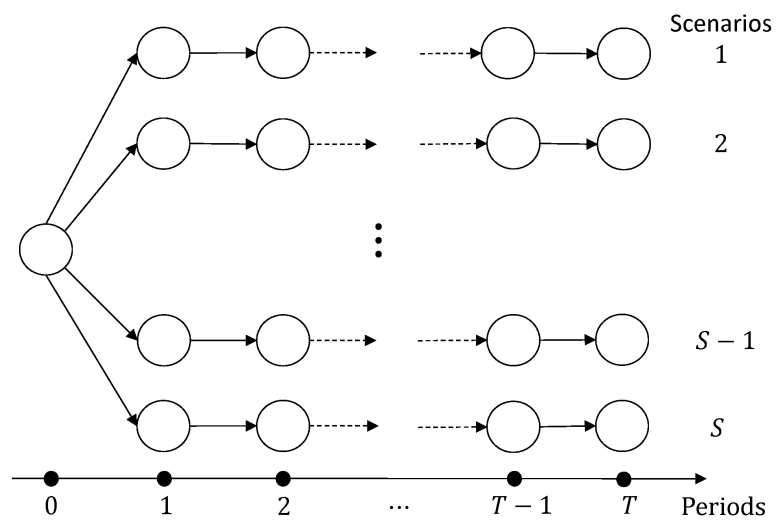

The K-means method aggregates the scenario fan into a scenario tree with a smaller number of nodes by bundling the nodes in each period into clusters. The process of the K-means method is described in Figure 5. The scenario tree has characteristics of a branching structure and non-anticipativity. Non-anticipativity means that decision-makers cannot make decisions in anticipation of branching into different scenarios in the future. If two scenarios $s 1, s 2$ satisfy the condition $\left(\xi_{1}^{s 1}, \ldots, \xi_{t}^{s 1}\right)=$ $\left(\xi_{1}^{s 2}, \ldots, \xi_{t}^{s 2}\right)$ for some period $t$, they are indistinguishable up to period $t$. The decisions made for scenario $s 1$ up to period $t$ must be the same as those made for $s 2$ up to period $t$. 
Figure 5 Clustering process of the K-means method considering rental quantity

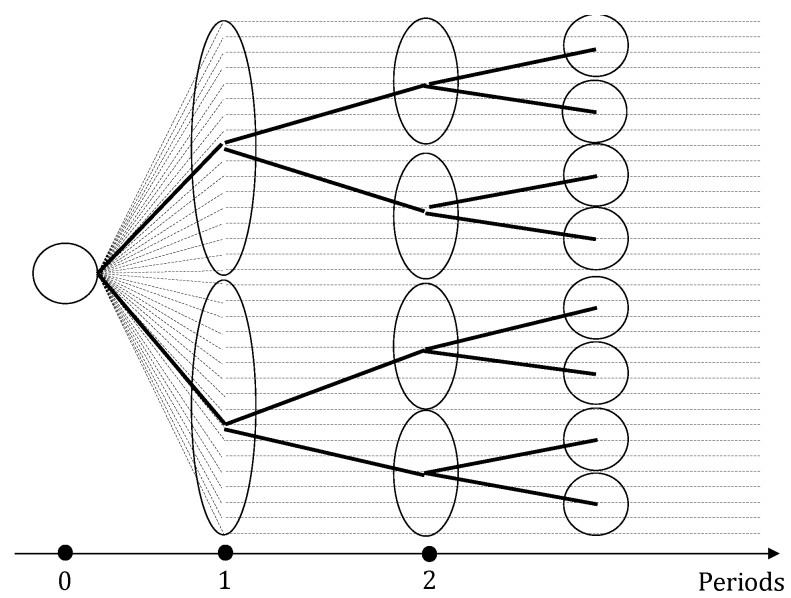

The notations and algorithm of the K-means method are as follows:

\section{Notations}

$T$ the number of stages

$S$ the number of scenarios in a scenario fan

$K_{t} \quad$ the number of clusters in stage $t$

$b_{t} \quad$ the number of son nodes branching from each node in stage $t$

$\xi_{t}^{s} \quad$ rental quantity in stage $t$ under scenario $s,(s=1, \ldots, S)$

$\bar{\xi}^{i} \quad$ centroid value of cluster $i$

$p_{t}^{k} \quad$ occurrence probability of node (cluster) $k$ in stage $t$.

Algorithm of K-means method

Step $0 \quad$ Initialisation.

Set $t=0, k=1$. Start from node 1 in period 0 .

Step 1 Selection of initial centroid.

Using scenario $s$ aggregated to node $k$ in period $t$, randomly select $b_{t}$ scenarios out of $\xi_{t+1}^{s}$. Let these be the centroids $\bar{\xi}_{t+1}^{i}$ of cluster $i$, $\left(i=1, \ldots, b_{t}\right)$.

Step 2 Cluster assignment.

Assign $\xi_{t+1}^{s}(s=1, \ldots, S)$ to the cluster $i\left(i=1, \ldots, b_{t}\right)$, such that centroid $\bar{\xi}_{t+1}^{i}$ is nearest to $\xi_{t+1}^{s}$. 
Step 3 Centroid's update.

Let new centroid values $\bar{\xi}_{t+1}^{i}$ be the average of scenarios assigned to cluster $i$.

Step 4 Convergence test.

Go to Step 2 until convergence. The termination criteria of convergence is that no scenarios are assigned to different clusters.

Step 5 Calculation of probability.

Let probability of $\bar{\xi}_{t+1}^{i}$ be the sum of probability of scenarios $\xi_{t+1}^{s}$ assigned to cluster $i$.

Step 6 Termination test.

If $k<K_{t}$, let $k_{t}=k_{t}+1$, go to Step2.

Otherwise, let $t=t+1$, if $t=T$, stop. Else let $k=1$, go to Step 2 .

\subsection{Modification of return quantity}

Using aggregated scenarios through the K-means method, we need to calculate the return quantity for it to be consistent with the rental quantity. If the return quantity of node $k$ at stage $t$ is calculated using scenarios aggregated to node $k$ at stage $t$, the rental and return quantities become different and not consistent. This example is shown in Figure 6. Calculating the centroids of each cluster, rental quantity $\bar{\xi}_{t-1}$ does not coincide with return quantity $\bar{\zeta}_{t}$. The rental quantity in period $t-1$ is 100 , but the return quantity in period $t$ is 150 and 75. Therefore, using scenarios aggregated to node $k$ at stage $t$, we calculate the return quantity of descendant nodes after stage $t+1$ (Figure 7).

Figure 6 An example of inconsistent rentals and returns

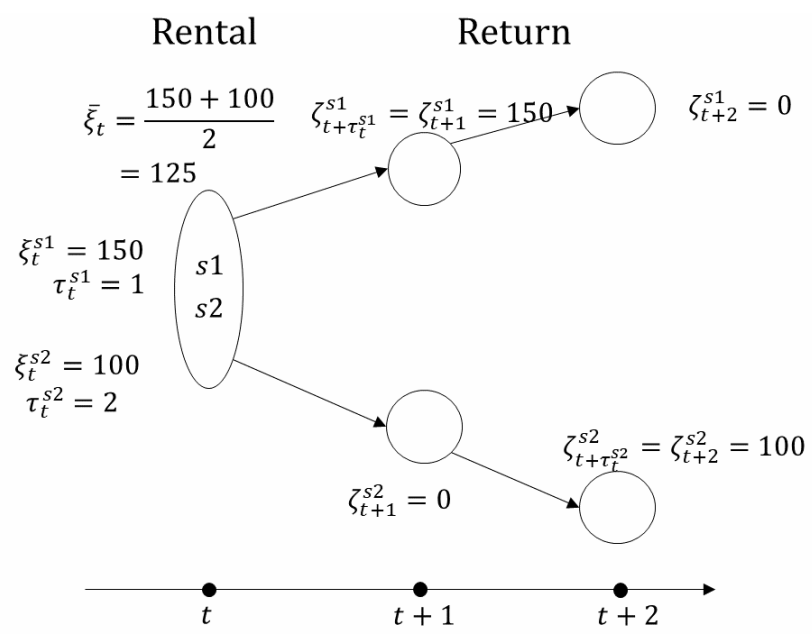


Figure 7 Modification of return quantity after stage $t+1$

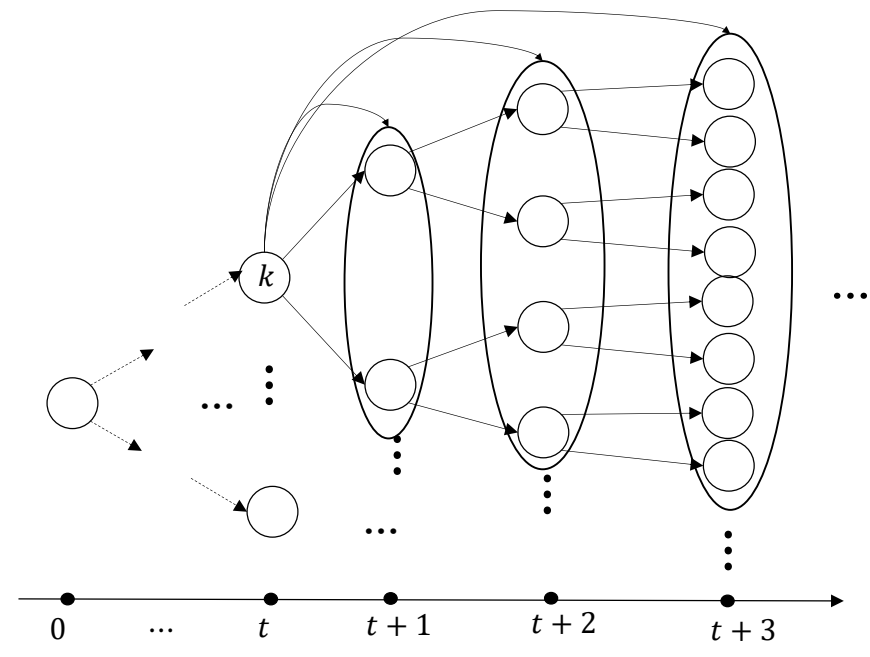

The notations and algorithm of the calculation of the return quantity are as follows.

Figure 7 represents Step 1 in the algorithm.

\section{Notations}

$D_{\tau}(k, t) \quad$ The set of descendant nodes in stage $\tau$ for node $k$ in stage $t$

$C_{t}^{k} \quad$ The set of scenarios aggregated to node $k$ in stage $t$

$p^{s} \quad$ Probability of scenario $s$ in scenario fan

$\zeta_{t}^{s} \quad$ Original return quantity in stage $t$ under scenario $s$ of scenario fan

$\bar{\zeta}_{t}^{l} \quad$ Aggregated return quantity of node $l$ in stage $t$ of scenario tree

Computational algorithm of return products

Step 0 Initialisation.

Set $t=1, k=1$

Step 1 Update of return quantity.

Calculate return quantity $\bar{\zeta}_{\tau}^{l}$ after period $t+1$ for all $l$ in $D_{\tau}(k, t)$, $\tau=t+1, \ldots, T$, using return quantity $\zeta_{\tau}^{s}(\tau=t+1, \ldots, T)$ for rental quantity $\xi_{t}^{s}$ of scenarios aggregated to node $k$ in stage $t$.

$\bar{\zeta}_{\tau}^{l} \leftarrow \bar{\zeta}_{\tau}^{l}+\sum_{s \in C_{t}^{k}} p^{s} \zeta_{\tau}^{s}, \quad \tau=t+1, \ldots, T, \forall l \in D_{\tau}(k, t)$

Step 2 Termination test.

If $k<K_{t}$, let $k_{t}=k_{t}+1$, go to Step 1 .

Otherwise, let $t=t+1$, if $t=T$, stop. Else, let $k=1$, go to Step 1 . 
The scenario tree of Figure 6 is modified as shown in Figure 8.

Figure 8 Modification of return quantity

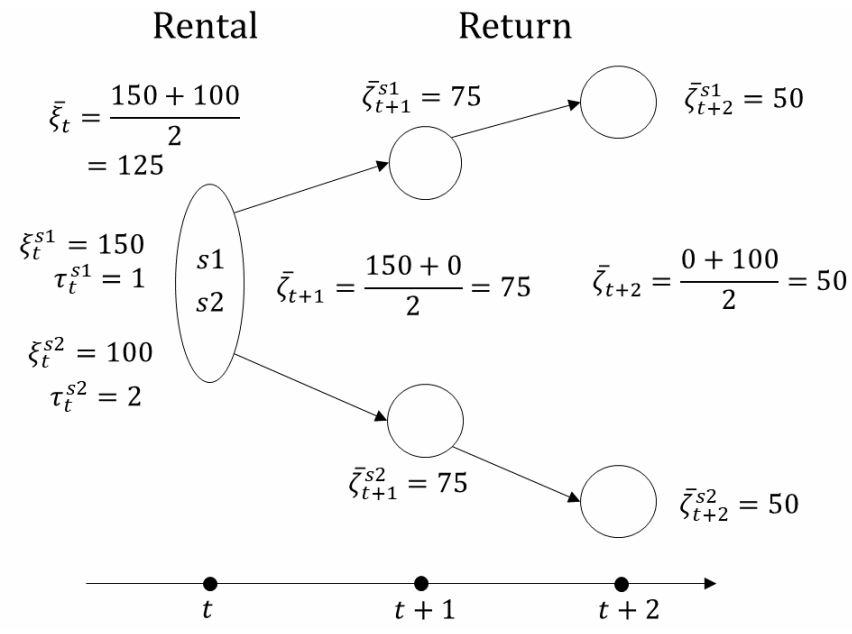

\section{Numerical experiments}

We demonstrate the effectiveness of the proposed model using numerical experiments. The computer used for this experiment has a Core i7-7700 CPU with 32 GB memory and uses AMPL-CPLEX version 12.6.2.0 on Windows 10 Pro. The problems were described using AMPL and solved by CPLEX directly.

The locations of existing factories, potential factories, and bases are generated in a uniform pattern on a $[0,100] \times[0,100]$ grid. The order cost $R_{f i t}^{k}$ of base $i$ for factory $f$ is defined as $(1+0.1 \times$ the distance between the factory and the base). The lateral transshipment cost $L_{i j t}^{k}$ from base $i$ to base $j$ is defined as $(0.5 \times$ the distance between the bases). Values of major parameters are as follows.

As mentioned in the previous section, the demand variation of the rental products was represented by a scenario tree. In addition, we assume long-term trends in the demand (Morikawa and Nakamura, 2000) of rental products. For example, the demand of unit houses increases before big projects. On the contrary, the demand decreases after the projects. Many big projects are planned in Japan. Therefore, decision making before and after the projects is assumed. Three trends are assumed: flat, growth, and decline. In the growth trend, demand increases over time. The rental quantity $\tilde{\xi}_{t}$ in period $t$ in the growth trend follows a normal distribution with a mean of $(100+12 t)$ and variance of 10 . In the decline trend, demand declines over time. The rental quantity $\tilde{\xi}_{t}$ in period $t$ in the decline trend follows a normal distribution with a mean of $(100-10 t)$ and variance of 10. This can support decision making considering future market trends.

There are many studies with models that consider only growth trends. As of 2020, the global economy is in recession due to COVID-19. Therefore, analysing multiple trend variants is more important than before. More case studies should be analysed in the future. 
Table 1 Values of major parameters

\begin{tabular}{lcc}
\hline Notation & & Value \\
\hline The number of periods & $T$ & 6 \\
The number of scenarios in a scenario fan & $S$ & 1280 \\
The number of existing factories & $E$ & 1 \\
The number of potential factories & $C$ & 3 \\
The number of bases & $I$ & 20 \\
The number of customers belonging to base $i$ & $N_{i}$ & 6 \\
Capacity of factory $f$ & $C A P_{f}$ & 850 \\
Installation cost of potential factory $f \in C$ & $F_{f t}$ & $100,000 *\{1-0.1 *(t-1)\}$ \\
in the period $t$ & & 4 \\
Inventory cost & $H_{i t}^{k}$ & 10 \\
Stockout cost & $P_{i t}^{k}$ &
\end{tabular}

The value of the stochastic solution (VSS) (Birge, 1997; Kall and Wallace, 1994) is used to verify the effectiveness of a stochastic programming model. The deterministic model is formulated by replacing random variables with the mean of all scenarios. Let EEV be the optimal objective value of the stochastic programming problem with the optimal solution of the deterministic problem. Let RP be the optimal objective value of the stochastic programming problem. VSS is defined as follows using EEV and RP.

$$
V S S=E E V-R P
$$

Table 2 presents the results of comparative experiments of RP and EEV. The improvement rate of the cost using a stochastic model is defined as follows.

$$
\text { Improvement rate }(\%)=\frac{V S S}{E E V} \times 100
$$

In the stochastic model (RP), a better optimal value is obtained. The improvement rate of the decline trend is the highest because a new factory is not installed in the stochastic model (RP). In the flat and growth trends, the solutions of RP and EEV are equal because the decisions on facility location are identical. To avoid the risk of stockout, it is necessary to set the value of $\gamma_{t}$ low. Constraint (9) also limits the sum of the values of the variable $y_{i t}^{k}$. In order to satisfy constraints (7) and (8), the value of the variable $w_{f \tau}$ in constraint (4) becomes 1 and a new facility must be installed. In particular, we consider the decline case. When solving RP, multiple scenarios are dealt with simultaneously to minimise the expected cost. Since the level of demand is low in the decline case, demands are barely met in many scenarios. Even if new equipment is not introduced, the solution that the out-of-stock probability is below $\gamma_{t}$ can be obtained. However, when solving VSS, a deterministic problem based on expected values that has an average scenario as the only scenario is solved. In the decline case, most of the scenarios have low demand and close values. However, if the demand is a little high in a specific scenario, it is necessary to introduce a new facility to meet the expected demand. In our model, the first stage decision is limited to the installation of new facilities. In the flat and growth trends, the optimal decisions are to install a new facility. However, in the decline trend, the stochastic model (RP) can avoid these expensive costs. The effectiveness of a stochastic model was shown considering demand trends. The calculation time was 1,000 seconds in the flat trend and limited to 40,000 seconds 
in the growth and the decline periods. The number of decision variables is 2,500 for $0-1$ variables and 50,000 for continuous variables, and the number of constraints is about 10,000 .

Table 2 Evaluation of solution for stochastic model $\left(\gamma_{t}=0.05\right)$

\begin{tabular}{lcccc}
\hline Demand trend & $R P$ & EEV & VSS & Improvement rate (\%) \\
\hline growth & 162,084 & 162,084 & 0 & 0 \\
flat & 139,426 & 139,426 & 0 & 0 \\
decline & 45,246 & 127,397 & 82,151 & 64.5 \\
\hline
\end{tabular}

Table 3 compares costs to show the effectiveness of lateral transshipment between bases. Let $\mathrm{GAP}(\%)$ be the reduction rate of costs by performing a lateral transshipment. $\mathrm{GAP}(\%)$ is calculated as follows.

$$
\operatorname{GAP}(\%)=\frac{B-A}{B} \times 100
$$

In the case of allowing lateral transshipment, a better optimal value is obtained. In the decline trend, the cost is significantly reduced as a new factory is not installed by lateral transshipment. If lateral transshipment is prohibited, the new factory must be installed due to service level constraints (7), (8), and (9). The effectiveness of a lateral transshipment was demonstrated by the comparative experiment.

Table 3 Evaluation of lateral transshipment $\left(\gamma_{t}=0.05\right)$

\begin{tabular}{lccc}
\hline & $A$ & $B$ & \\
& Lateral transshipment is available & Not available & GAP (\%) \\
\hline growth & 162,084 & 166,047 & 2.4 \\
flat & 139,427 & 140,688 & 0.9 \\
decline & 45,246 & 128,292 & 64.7 \\
\hline
\end{tabular}

Table 4 presents a comparison of costs when service level requirement for stockout $\gamma_{t}$ varies. As stockout rate $\gamma_{t}$ increases, the cost decreases. By increasing the service level requirement regarding stockout $\gamma_{t}$, the inventory and the order quantity are reduced. By setting $\gamma_{t}$ high, the cost is significantly reduced because new factories are not installed. It is possible to manage supply chains efficiently by controlling service level. In the growth and the flat case, a new facility was installed at 0.05 , but no new facility was installed at 0.075 . Due to the large installation cost of the facility, there was a large difference in the total cost.

Table 4 Comparison by stockout rate

\begin{tabular}{lccccc}
\hline stockout rate & 0 & 0.025 & 0.05 & 0.075 & 0.1 \\
\hline growth & 165,467 & 163,329 & 162,084 & 106,319 & 104,771 \\
flat & 139,431 & 139,426 & 139,426 & 69,246 & 68,378 \\
decline & 127,397 & 127,397 & 45,246 & 44,569 & 43,942 \\
\hline
\end{tabular}




\section{Conclusions}

In this study, we proposed a multi-period stochastic model for a lateral transshipment problem of rental products. A scenario tree was constructed using the K-means method considering uncertain rentals and returns in a multi-period. The future economic situation was considered by introducing demand trends. Numerical experiments revealed the effectiveness of the proposed model. The effectiveness of a stochastic model was shown by the VSS. The cost was reduced by lateral transshipment between bases. By controlling the upper limit of the stockout rate, it became possible to make decisions according to various service levels.

In a future study, we would like to introduce production and transportation lead times in the model, which would make the proposed model more realistic. Since the proposed model is a large scale 0-1 mixed integer programming problem, an efficient solution is needed.

\section{References}

Agrawal, V., Chao, X. and Seshadri, S. (2004) 'Dynamic balancing of inventory in supply chains', European Journal of Operational Research, Vol. 159, No. 2, pp.296-317.

Birge, J.R. (1997) 'Stochastic programming computation and applications', INFORMS Journal on Computing, Vol. 9, No. 2, pp.111-133.

Birge, J.T. and Louveaux, F. (1997) Introduction to Stochastic Programming, Springer-Verlag, New York.

Gülpınar, N., Rustem, B. and Settergren, R. (2004) 'Simulation and optimization approaches to scenario tree generation', Journal of Economic Dynamics and Control, Vol. 28, No. 7, pp.1291-1315.

Høyland, K. and Wallace, S.W. (2001) 'Generating scenario trees for multistage decision problems', Management Science, Vol. 47, No. 2, pp.295-307.

$\mathrm{Hu}, \mathrm{Z}$. and $\mathrm{Hu}, \mathrm{G}$. (2016) 'A two-stage stochastic programming model for lot-sizing and scheduling under uncertainty', International Journal of Production Economics, Vol. 180, pp.198-207.

Kall, P. and Wallace, S.W. (1994) Stochastic Programming, John Wiley \& Sons, Chichester.

Kaut, M. and Wallace, S.W. (2007) 'Evaluation of scenario generation methods for stochastic programming', Pacific Journal of Optimization, Vol. 3, No. 2, pp.257-271.

Kiya, F. and Davoudpour, H. (2012) 'Stochastic programming approach to redesigning a warehouse network under uncertainly', Transportation Research Part E, Vol. 48, No. 5, pp.919-936.

Laporte, G. and Louveaux, F.V. (1993) 'The integer L-shaped method for stochastic integer programs with complete recourse', Operations Research Letters, Vol. 13, No. 3, pp.133-142.

Lin, C.K.Y. (2009) 'Stochastic single-source capacitated facility location model with service level requirements', Int. J. Production Economics, Vol. 117, No. 2, pp.439-451.

Melachrinoudis, E. and Min, H. (2000) 'The dynamic relocation and phase-out of a hybrid, two-echelon plant/warehousing facility: a multiple objective approach', European Journal of Operational Research, Vol. 123, No. 1, pp.1-15.

Melachrinoudis, E., and Min, H. (2007) 'Redesigning a warehouse network', European Journal of Operational Research, Vol. 176, No. 1, pp.210-229.

Melo, M., Nickel, S. and da Gama, F.S. (2005) 'Dynamic multi-commodity capacitated facility location: a mathematical modeling framework for strategic supply chain planning', Computers and Operations Research, Vol. 33, No. 1, pp.181-208. 
Möller, A., Römisch, W. and Weber, K. (2004) 'A new approach to O\&D revenue management based on scenario trees', Journal of Revenue \& Pricing Management, Vol. 3, No. 3, pp.265-276.

Morikawa, K. and Nakamura, S. (2000) 'Comparative study of two production planning models under demand uncertainty', Japanese Industrial Management Association, in Japanese, Vol. 51, No. 3, pp.227-236.

Naderi, S., Kilic, K. and Dasci, A. (2020) 'A deterministic model for the transshipment problem of a fast fashion retailer under capacity constraints', International Journal of Production Economics, Vol. 227, Article No. 107687, p.107687.

Paterson, C., Kiesmüller, G., Teunter, R. and Glazebrook, K. (2011) 'Inventory models with lateral transshipments: a review', European Journal of Operational Research, Vol. 210, No. 2, pp.125-136.

Schildbach, G. and Morari, M. (2016) 'Scenario-based model predictive control for multi-echelon supply chain management', European Journal of Operational Research, Vol. 252, No. 2, pp.540-549.

Shiina, T., Umeda, M., Imaizumi, J., Morito, S. and Xu, C. (2014) 'Inventory distribution problem via stochastic programming', Asian Journal of Management Science and Applications, Vol. 1, No. 3, pp.261-277.

Shiina, T. and Birge, J.R. (2002) 'Multistage stochastic programming model for electric power capacity expansion problem', Japan Journal of Industrial and Applied Mathematics, Vol. 20, No. 3, pp.379-397.

Shiina, T. (2015) Stochastic Programming, in Japanese, Asakura Syoten, Tokyo.

Šutiene, K. Makckas, D. and Pranevičius, H. (2010) 'Multistage K-means clustering for scenario tree construction', Informatica, Vol. 21, No. 1, pp.123-138.

Topan, E. and van der Heijden, M.C. (2020) 'Operational level planning of a multi-item two-echelon spare parts inventory system with reactive and proactive interventions', European Journal of Operational Research, Vol. 284, No. 1, pp.164-175.

Van Slyke, R. and Wets, R.J-B. (1969) 'L-shaped linear programs with applications to optimal control and stochastic linear programs', SIAM Journal on Applied Mathematics, Vol. 17, No. 4, pp.638-663.

Xu, D., Chen, Z. and Yang, L. (2012) 'Scenario tree generation approaches using K-means and LP moment matching methods', Computational and Applied Mathematics, Vol. 236, No. 17, pp.4561-4579. 